\title{
Familiarity does not affect the unilateral field advantage for repetition detection
}

\author{
Serena J. Butcher • Patrick Cavanagh
}

Published online: 25 April 2012

(C) Psychonomic Society, Inc. 2012

\begin{abstract}
We have previously reported evidence that repetitions of letters, colors, sizes, and common motion paths are more rapidly detected when they are presented unilaterally (i.e., both in the same visual field) versus bilaterally (one element in each visual field; Butcher and Cavanagh (Attention, Perception, \& Psychophysics 70:714-724, 2008). Here, we report evidence that this unilateral field advantage (UFA) for repetition detection does not depend on prior experience with the elements that comprise the repetition. In Experiment 1, native English, Persian, and Japanese speakers were tested on a repetition detection task involving characters from Western, Arabic, and Japanese character sets. The character sets were tested in blocks, in each of which subjects were presented with four characters for $16 \mathrm{~ms}$ and asked to report whether any two of the characters were identical. The subjects were faster detecting repetitions that were presented unilaterally rather than bilaterally, and there was no interaction with stimulus familiarity. A second experiment replicated this finding with native English speakers only, using a longer stimulus duration $(150 \mathrm{~ms})$. We had previously proposed that the UFA arises because the low-level processes that group physically identical items operate more efficiently within than across hemifields. Our data now indicate that this grouping process is insensitive to item familiarity, supporting the claim that the process is low-level.
\end{abstract}

Keyword Unilateral field advantage · Repetition effects . Grouping $\cdot$ Segmentation

\footnotetext{
S. J. Butcher $(\bowtie) \cdot$ P. Cavanagh

Department of Psychology, Harvard University,

Cambridge, MA, USA

e-mail: Sbutcher@hamilton.edu
}

The visual system is shaped by experience in the visual world. Congenitally and early blind individuals who recover sight later in life have difficulty with the 3-D interpretation of line drawings, depth judgments, transparency, face recognition, and object recognition (Bouvrie \& Sinha, 2007; Fine et al., 2003; Ostrovsky, Andalman, \& Sinha, 2007). For normally sighted individuals, extended experience with particular stimuli imparts the many processing advantages that accrue to familiar items. The effects of familiarity have been investigated in many studies, in which it has typically been shown that familiarity benefits performance. However, here we report evidence from a repetition detection task in which familiarity provided no benefit, suggesting that the process for detecting repetitions operates at a very early level of visual analysis, before the advantages of familiarity are available. Processing speed is affected by whether the repeated elements are presented unilaterally - that is, both in one visual field - versus bilaterally - with one element in each visual field - and this difference is the same for both familiar and unfamiliar stimuli.

The role of familiarity in visual search tasks has been explored frequently. Wang, Cavanagh, and Green (1994) manipulated stimulus familiarity by having subjects search for regular or mirror-reversed letter targets among regular or mirror-reversed distractors. Familiar distractors (regular letters) produced parallel search for unfamiliar targets (mirrorreversed letters). Wang et al. interpreted these results as suggesting that the familiarity of individual items is extracted rapidly from the search displays, allowing the familiar background to be grouped quickly and the unfamiliar target to be efficiently detected. Malinowski and Hübner (2001) investigated the effect of familiarity on visual search in German and Slavic subjects, using an "N" and a mirrorreversed "N" as the target and distractor pairs. For German subjects, "N" was a familiar letter, whereas for Slavic 
subjects, both "N" and the mirror-reversed "N" were familiar letters. For German subjects, search for the familiar "N" target among unfamiliar mirror-reversed " $\mathrm{N}$ " distractors was inefficient, producing a search slope of $23 \mathrm{~ms} /$ item. Search for the unfamiliar mirror-reversed target among familiar distractors, however, was very efficient (slope $=-0.6 \mathrm{~ms} /$ item). These results are similar to those of Wang et al. However, the search slopes from Slavic participants were efficient for both combinations of "N"/mirror-"N" target/distractor pairs (search slopes of $-1.2 \mathrm{~ms} /$ item and $0.5 \mathrm{~ms} /$ item, respectively). As Slavic subjects were familiar with both "N" and mirror-"N," this result indicates that efficient search can occur for familiar targets among familiar distractors.

Rauschenberger and Yantis (2006), using a paradigm originally developed by Treisman (Treisman, 1986; Treisman \& Gormican, 1988; Treisman \& Souther, 1985), found that subjects were more efficient at detecting a circle with a vertical line among circles without lines (i.e., $\Psi$ among $\bigcirc$ s) than at detecting the target in the reverse arrangement ( $\bigcirc$ among $\bigcirc$ s), replicating Treisman's original findings. Rauschenberger and Yantis also tested the same subjects' performance on detecting a circle with a 45-deg line, a closer replication of a Western "Q" among "O"s. They found that search became even more efficient for this arguably more familiar target, even though the low-level features of the stimuli were the same in both conditions.

Critically, Rauschenberger and Chu (2006) also ran both search tasks on rural Chinese participants, who had no previous experience with the Western alphabet. The Chinese subjects replicated the basic search asymmetry: They were faster searching for a "Q" among "O"s than for an "O" among "Q"s. However, there was no effect of the orientation of the small bar; the Chinese subjects were equally fast finding the $Q$ with the vertical line and the "Q" with the 45-deg line. This finding suggests that when subjects are familiar with the shape of the stimulus, as with "Q," a search benefit results from presenting it in its canonical orientation.

Shen and Reingold (2001) investigated the role of familiarity in visual search for familiar and novel Chinese symbols in native English and native Chinese speakers. Both groups were tested in two search tasks. In the first task, the target was a Chinese character 古, meaning "ancient or old," among 모s, a meaningless character comprised of a rearrangement of the familiar characters' features. In the second task, the target and distractor statuses were reversed for each character. For Chinese speakers, the first task was a search for a familiar target among unfamiliar distractors, and the second a search for an unfamiliar target among familiar distractors. For English speakers, both tasks were searches for unfamiliar targets among unfamiliar distractors. The Chinese participants showed a search asymmetry. They were faster searching for the unfamiliar target 모 among familiar distractors 古, with a search slope of $33 \mathrm{~ms} /$ item, than searching for the familiar target 古 in the unfamiliar distractors 무 (search slope $=57 \mathrm{~ms} /$ item). The English speakers, on the other hand, showed no search asymmetry: They were equally fast at both tasks. For 只among 古 s, the search slope was $24 \mathrm{~ms} / \mathrm{item}$, and for 古 among 只s, the search slope was $19 \mathrm{~ms} /$ item. For Chinese subjects, distractor familiarity speeded search more than did target familiarity; however, search slopes were steep even in the faster familiar-distractor condition.

We (Butcher \& Cavanagh, 2008), along with others (Hayes, Swallow, \& Jiang, 2010; Weissman, Banich, \& Puente, 2001), have reported evidence of a unilateral field advantage (UFA) for detecting repeated elements. In our work, subjects were faster at detecting repetitions of letters, colors, sized disks, and common motion paths when the stimuli were presented unilaterally to either the left or the right visual field versus bilaterally, with one element of the repetition in each visual field. This reaction time benefit ranged from approximately $30 \mathrm{~ms}$, for repeated sizes, to $100 \mathrm{~ms}$, for repeated letters.

We proposed that the detection of physical repetitions relies on a low-level grouping process. This process operates more efficiently within a hemifield than across hemifields, producing a UFA for repetition detection. The UFA occurs for both static and moving repetitions (Butcher \& Cavanagh, 2008). Thus, it seems to reflect a general lowlevel perceptual-grouping benefit for items within the same hemifield. We found no systematic differences between detecting repetitions in the left versus the right visual field, providing further evidence that this benefit reflects a stage of processing that occurs before hemispheric functional specialization.

The purpose of the present set of experiments was to investigate whether the familiarity effects that are ubiquitous in visual search tasks can also be found for repetition detection. In this way, we sought to use the presence or absence of a familiarity effect as a litmus test for higher-level visual processing. We are using the term "higher-level" in this context to connote a level of processing that is affected by prior exposure. To foreshadow the results, we did not find that familiarity affected the UFA for repetition detection. This result strengthens our previous claims (Butcher \& Cavanagh, 2008) that the advantage arises from a lowlevel grouping process. Furthermore, it demonstrates that this process can operate equally efficiently on novel and familiar items within a hemifield, based only on the featural similarity of the items.

In both our previous work on the UFA and that of others on visual field effects, the subjects were familiar with all of 
the stimulus classes tested. Common stimuli in these experiments include Western alphabetic characters (Banich \& Belger, 1990; Butcher \& Cavanagh, 2008; Cavanagh \& Parkman, 1972; Hayes et al., 2010; Weissman \& Banich, 1999; Weissman et al., 2001), colors (Butcher \& Cavanagh, 2008; Hayes et al., 2010), and objects (Hayes et al., 2010). The use of these highly familiar stimuli has left open the possibility that prior exposure to the elements comprising the repetition is necessary to produce a UFA for repetition detection.

In visual search tasks that produce familiarity effects, subjects are often presented with familiar and unfamiliar items in the same display (Rauschenberger \& Chu, 2006; Shen \& Reingold, 2001; Wang et al., 1994). Thus, in all displays the subjects are familiar with a least one item present. In our paradigm, subjects were presented with either all familiar or all unfamiliar stimuli, and we did not find effects of item familiarity. This indicates that familiarity is not necessary to produce the UFA and does not moderate the effect.

Here, in Experiment 1, using familiar and unfamiliar characters and brief presentation $(16 \mathrm{~ms})$, we show that stimulus familiarity did not affect the UFA for repetition detection. That is, fast unilateral repetition detection occurred to the same extent for both familiar and novel elements; familiarity did not interact with the UFA. In Experiment 2, we show that at longer stimulus durations $(150 \mathrm{~ms})$, we still found no effect of familiarity on the UFA for repetition detection. This finding supports our theory that the UFA reflects a general low-level perceptual-grouping process that is efficient within a hemifield.

\section{Experiment 1}

\section{Method}

Subjects Nine subjects, including three native English speakers (two female and one male, median age 24), three native Farsi speakers (one female and two male, median age 25 ), and three native Japanese speakers (one female and two male, median age 21) participated in this experiment and were paid $\$ 10$ per hour for their participation. All of the subjects had some level of exposure to the Western alphabet: The native Persian speakers were graduate students at Harvard University, and the native Japanese speakers were currently participating in an English-as-a-second-language program in Cambridge, Massachusetts. The Farsi speakers were unfamiliar with Japanese characters, and the Japanese speakers were unfamiliar with the Persian characters. The English subjects were unfamiliar with both Arabic script (used with Farsi) and Japanese characters. Estimates of familiarity with the character sets were based on the subjects' self-reports. All subjects had normal or corrected-tonormal vision.
Stimuli The stimuli for the experiment were characters from the Western, Arabic, and katakana (Japanese) alphabets. Each block of trials used the characters from one alphabet. The character sets were scaled such that the largest characters in each set were approximately equivalent: The largest Western and Japanese characters subtended $1.5 \times 1.5 \mathrm{deg}$, and the largest Persian character subtended $1.8 \times 1.8 \mathrm{deg}$. Characters in any set with physical similarity to characters in another set were not included. The Western alphabetic set included 25 characters, the Arabic set included 28 characters, and the Japanese katakana set included 44 out of a possible 46 characters (two characters were not included due to their similarity with the Western uppercase "E" and "I").

Apparatus All stimuli were presented by a Macintosh computer on a 17-in. Apple Studio Display monitor at a resolution of $1,280 \times 1,024$ and a refresh rate of $60 \mathrm{~Hz}$ and were generated with the Vision Shell PPC graphics libraries (Comtois, 2003).

Procedure For all trials, the subjects' task was to determine whether two identical elements were present in a display of four items. On target-present trials, two out of the four items were identical, and on target-absent trials, all four items were unique. The four search items always appeared in the same positions during the experiment. The four search positions formed the vertices of an invisible $5 \times 5 \mathrm{deg}$ square around fixation. On each block of trials, the subjects were tested with one set of characters: Western (uppercase), Arabic, or Japanese (Fig. 1). The target letters were selected randomly, and all subjects were tested on each type of character. The order of block presentation was counterbalanced across subjects, with subjects performing 20 practice and 400 experimental trials for each block. A repeated character target was presented on $50 \%$ of the trials.

The subjects were instructed to press one of two keys as quickly as possible to indicate whether the target (a repeated character) was present or absent (all four characters were unique). Repeated characters, if present, could appear at one of four possible locations in the search quartet: (1) top (bilateral upper visual field), (2) bottom (bilateral lower visual field), (3) left visual field (unilateral), or (4) right visual field (unilateral). Repetitions were never presented on a diagonal, to ensure equidistant presentation of bilateral and unilateral targets. The position for the repetition was selected randomly on each trial, and all repetition positions were used with the same frequency.

Each trial was initiated by a subject's keypress. A fixation point was shown at the center of the screen for $200 \mathrm{~ms}$, and the start of each trial was preceded by a tone. The display was presented for $16 \mathrm{~ms}$, or one video frame. The subjects indicated the presence or absence of a repeated element via the computer keyboard, pressing the single-quote key (') for a repetition and 
Fig. 1 Schematic of the paradigm used for repetition detection. The left panels show Western characters, the middle panels Arabic characters, and the right panels Japanese katakana characters. The characters were presented in blocks. Native English, Persian, and Japanese speakers were tested, with all of them were tested in all blocks $(n=9)$

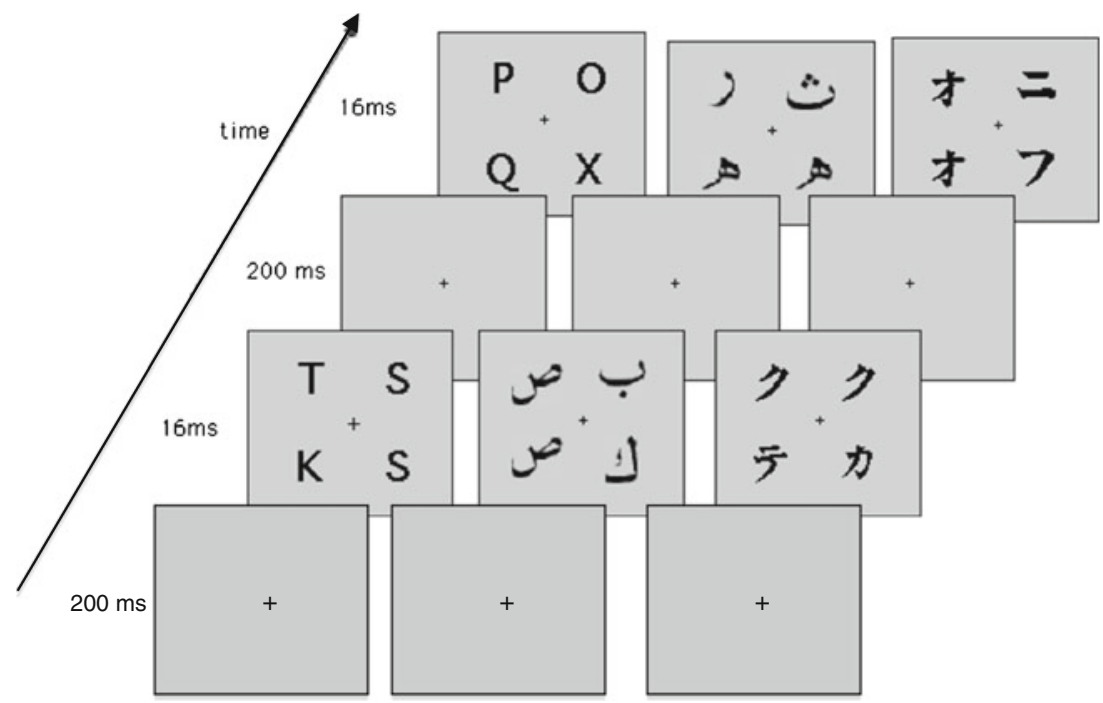

the "A" key for no repetition. Subjects were instructed to respond as quickly as possible to the presence or absence of the target items, while maintaining fixation, and to minimize errors.

\section{Results}

The mean reaction times and error data (misses) were subjected to a $3 \times 2 \times 3$ repeated measures ANOVA with Character Type (Western, Arabic, or katakana) and Repeated Character Laterality (unilateral or bilateral) as withinsubjects factors and Native Language (English, Farsi, or Japanese) as a between-subjects factor (Table 1).

Figure 2 shows the mean reaction times for detecting character repetitions as a function of the repeated characters' laterality (unilateral or bilateral) for Western, Arabic, and Japanese (katakana) characters among the native English, Japanese, and Farsi speakers. The ANOVA revealed a significant main effect of laterality $[F(1,6)=46.88, p<$ $.0005]$ : For all characters tested across all subjects, unilateral repetitions were detected faster that bilateral repetitions, with differences ranging from 32 to $109 \mathrm{~ms}$ (Table 2).

We found a significant main effect of native language $[F(2$, $6)=5.75, p<.04]$. A Fisher's least-significant-difference analysis showed that the English subjects overall were faster than the Farsi subjects $(p<.02)$. No other pairwise comparisons were significant. Native language did not have a significant interaction with any other factor, and there was no main effect of character $[F(2,12)=0.54, p=.60]$.

The data reveled a significant interaction between laterality and character $[F(4,12)=4.21, p<.04]$. Figure 3 shows mean reaction times as a function of character, collapsed across all native languages. For Western characters, unilateral repetitions were detected $97 \mathrm{~ms}$ faster than bilateral repetitions $[t(8)=6.13, p<.0003]$. For Arabic characters, unilateral repetitions were detected $81 \mathrm{~ms}$ faster than

Table 1 Experiment 1 repetition detection: Repeated measures $2 \times 3 \times 3$ ANOVA-laterality (unilateral, bilateral) $\times$ character (Western, katakana, Arabic) $\times$ native language (English, Japanese, Persian)

\begin{tabular}{|c|c|c|c|c|c|}
\hline Source & Sum of Squares & $d f$ & Mean Square & $F$ & $p$ \\
\hline \multicolumn{6}{|l|}{ Within Subjects } \\
\hline Laterality & $79,412.79$ & 1 & $79,412.79$ & 46.88 & .0005 \\
\hline Laterality $\times$ Native Lang. & 508.17 & 2 & 254.09 & 0.15 & .86 \\
\hline Error (Laterality) & $10,164.66$ & 6 & $1,694.11$ & & \\
\hline Character & $13,657.66$ & 2 & $6,828.83$ & 0.54 & .60 \\
\hline Character $\times$ Native Lang. & $46,918.46$ & 4 & $11,729.62$ & 0.93 & .48 \\
\hline Error (Character) & $151,806.34$ & 12 & $12,650.53$ & & \\
\hline Laterality $\times$ Character & $4,919.98$ & 2 & $2,459.99$ & 4.21 & .04 \\
\hline Laterality $\times$ Character $\times$ Native Lang. & $1,781.77$ & 4 & 445.44 & 0.76 & .57 \\
\hline Error (Laterality $\times$ Character) & $7,003.66$ & 12 & 583.64 & & \\
\hline \multicolumn{6}{|l|}{ Between Subjects } \\
\hline Native Language & $328,065.88$ & 2 & $164,032.94$ & 5.75 & .04 \\
\hline Error (Native Language) & $171,059.56$ & 6 & $28,509.93$ & & \\
\hline
\end{tabular}



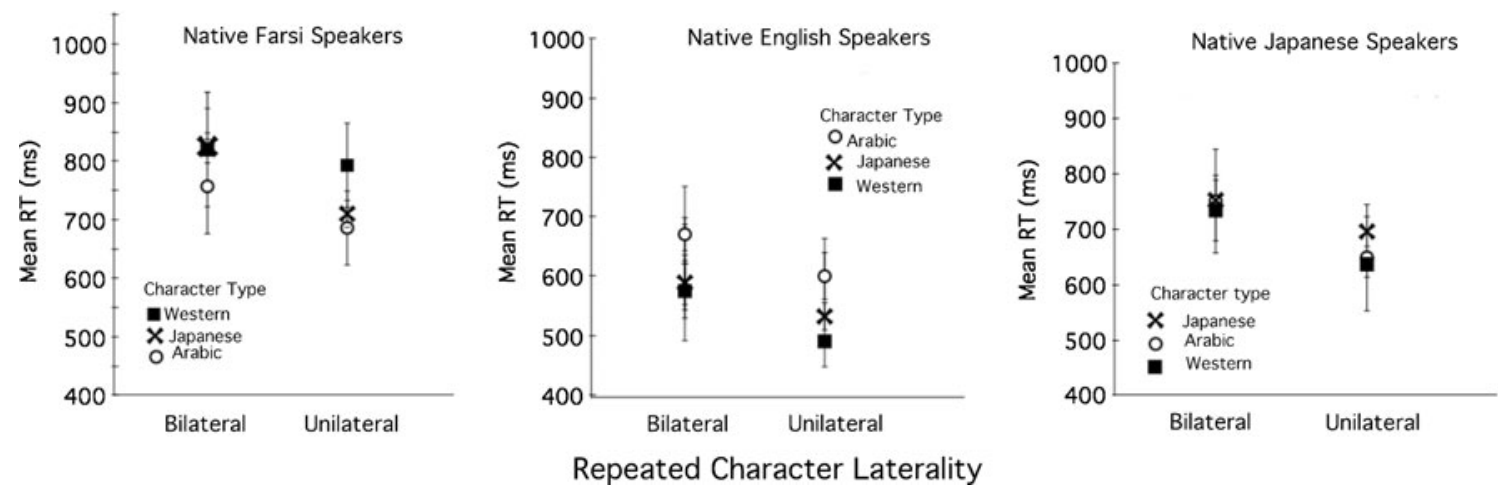

Fig. 2 Mean reaction times for native English, Farsi, and Japanese speakers for repetition detection with Western, Arabic, and katakana characters. The error bars indicate \pm 1 SEM

bilateral repetitions $[t(8)=5.58, p<.001]$. For Japanese characters, unilateral repetitions were detected $51 \mathrm{~ms}$ faster than bilateral repetitions $[t(8)=5.93, p<.0004]$.

Table 3 shows repetition detection miss rates as a function of character, native language, and missed target laterality. Though miss rates were low in all cells, the analysis revealed a significant main effect of laterality $[F(1,6)=$ 133.87, $p<.00003$ ] and a significant Laterality $\times$ Native Language interaction $[F(2,6)=6.85, p<.03]$. Native English and Japanese speakers made significantly fewer misses of unilateral repetitions [English, $t(2)=11.18, p<$ .0008 ; Japanese, $t(2)=5.98, p<.03$ ]. Native Farsi speakers showed no significant difference in miss rates as a function of laterality $[t(2)=3.06, p=.09)$. There were no main effects of character $[F(2,12)=1.93, p=.21]$ or native language $[F(2,6)=1.22, p=.36]$.

In the previous analysis, native language was used as an indirect metric of "familiarity," and it did not interact with any other variable in the reaction time analysis. The interaction in the error data was not indicative of a speed-accuracy trade-off.

We performed a second ANOVA (Table 4) to investigate further the possibility of a familiarity effect, with familiarity defined more broadly. In this analysis, familiarity was coded as a binary variable (familiar or unfamiliar) collapsed across all native languages. Native Farsi and Japanese speakers were considered to be familiar with Western characters and with their native language characters. Native English

Table 2 Unilateral field advantage (in milliseconds) for repetition detection as a function of native language and character type

\begin{tabular}{llll}
\hline Native Language & \multicolumn{3}{l}{ Character Type } \\
\cline { 2 - 4 } & Western & Arabic & Japanese \\
\hline English & 86 & 70 & 66 \\
Farsi & 109 & 71 & 32 \\
Japanese & 97 & 102 & 55 \\
\hline
\end{tabular}

Unilateral field advantage $=$ bilateral mean $\mathrm{RT}-$ unilateral mean RT speakers were considered to be familiar with Western characters only. These determinations were made on the basis of the self-reported familiarity assessment conducted before the experiment began. The mean reaction times of correct targetpresent responses and miss rates were each subjected to a within-subjects $2 \times 2$ ANOVA, with Target Laterality (bilateral or unilateral) and Character Familiarity (familiar or unfamiliar) as the factors. For the familiar and unfamiliar condition in this analysis, $n=9$.

Figure 4 shows reaction times as a function of laterality for familiar and unfamiliar characters, as defined for the second analysis. This analysis revealed a significant main effect of target laterality on reaction times $[F(1,8)=64.32$, $p<.00004$, two-tailed $]$ and on the error data $[F(1,8)=$ 54.66, $p<.00007$, two-tailed]. Unilateral repetitions, both familiar and unfamiliar, were detected faster than bilateral repetitions. The main effect of familiarity was not significant $[F(1,8)=1.70, p<.23]$, nor was the Familiarity $\times$ Laterality interaction $[F(1,8)=0.95, p<.36]$. Familiar unilateral

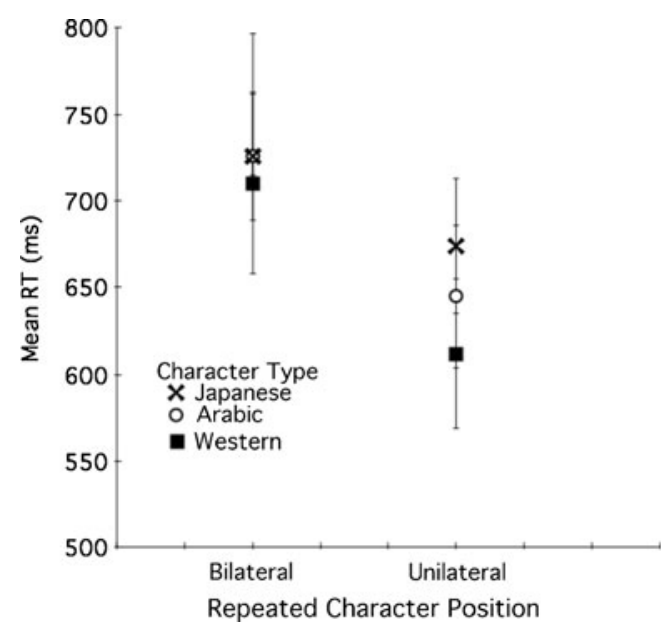

Fig. 3 Mean reaction times for Arabic, Japanese, and Western characters, collapsed across all native languages. For all character types, unilateral repetitions were detected significantly more quickly than bilateral repetitions (ranging from 51 to $97 \mathrm{~ms}$ at $p<.001$ or better, $n=9$ ) 
Table 3 Miss rate for repetition detection as a function of a missed target's laterality, for all characters by each subjects' native language

\begin{tabular}{|c|c|c|c|c|c|c|}
\hline \multirow[t]{3}{*}{ Native Language } & \multicolumn{6}{|c|}{ Character Type } \\
\hline & \multicolumn{2}{|l|}{ Western } & \multicolumn{2}{|l|}{ Arabic } & \multicolumn{2}{|l|}{ Japanese } \\
\hline & Bilateral & Unilateral & Bilateral & Unilateral & Bilateral & Unilateral \\
\hline English & $1.6 \%$ & $0.5 \%$ & $1.6 \%$ & $0.4 \%$ & $2.3 \%$ & $0.6 \%$ \\
\hline Farsi & $2.2 \%$ & $0.9 \%$ & $2.4 \%$ & $0.8 \%$ & $1.7 \%$ & $1.3 \%$ \\
\hline Japanese & $0.9 \%$ & $0.4 \%$ & $1.4 \%$ & $0.7 \%$ & $1.1 \%$ & $0.6 \%$ \\
\hline
\end{tabular}

targets were detected $88 \mathrm{~ms}$ faster than familiar bilateral targets $[t(8)=7.40, p<.0001]$, and unfamiliar unilateral targets were detected $69 \mathrm{~ms}$ faster than bilateral repetitions of unfamiliar characters $[t(8)=4.38, p<.002$, two-tailed]. Additionally, unilateral targets produced fewer misses than did bilateral targets. For familiar targets, the average miss rates were $1 \%$ for unilateral and $2 \%$ for bilateral $[t(8)=$ $4.79, p<.001$, two-tailed]. For unfamiliar targets, the average miss rates were $1 \%$ for unilateral and $3 \%$ for bilateral $[t$ $(8)=3.24, p<.01$, two-tailed]. The target-absent mean reaction times for familiar and unfamiliar trials were 781 and $825 \mathrm{~ms}$, respectively, and post-hoc testing revealed that this difference was not significant.

\section{Discussion}

The results of this experiment indicated that unilateral repetitions were detected faster than bilateral repetitions, regardless of stimulus familiarity. In the first analysis, familiarity was operationally defined on the basis of the speaker's native language, and native language did not interact with the unilateral field advantage. There was an interaction between native language and laterality in the error data; however, there was no evidence of a speedaccuracy trade-off. For two of the native languages, English and Japanese, the subjects were both faster and more accurate at detecting unilaterally presented repeated characters. For the third language, Farsi, there was no difference in miss rates between the unilateral and bilateral trials.
Our second analysis defined familiarity on the basis of exposure to the characters. We examined the possibility that a broader operational definition of familiarity would allow for more sensitivity to its potential effects in the data. However, again we did not find any significant interaction of familiarity effects or interactions with laterality. Unilateral repetitions were detected faster than bilateral repetitions for both familiar and unfamiliar characters. The 88-ms advantage for detecting unilateral versus bilateral repetitions was in the same range as the reaction time advantage that we had previously found for detecting familiar letter repetitions (Butcher \& Cavanagh, 2008).

In Experiment 1, we had used a brief stimulus presentation time (similar to that in Butcher \& Cavanagh, 2008) to prevent subjects from making saccadic eye movements to the target stimuli. This raised the question of whether our exposure times were sufficient to detect benefits from stimulus familiarity. ${ }^{1}$ Familiarity can be defined as link to memory from prior experience, and it is possible that additional viewing time might provide time to activate familiarity effects. In Experiment 2, we sought to investigate whether longer viewing times would reveal familiarity effects on reaction times or error rates for repetition detection.

\section{Experiment 2}

Method

Subjects Twelve undergraduates at Hamilton College (seven female and five male, median age 19) participated in

Table 4 Experiment 1, Analysis 2: Repeated measures $2 \times 2$ ANOVA—laterality (bilateral, unilateral) $\times$ character familiarity (yes, no)

\begin{tabular}{llllc}
\hline Source & Sum of Squares & $d f$ & Mean Square & $F$ \\
\hline Familiarity & $16,781.28$ & 1 & $16,781.58$ & .23 \\
Error (Familiarity) & $78,820.01$ & 8 & $9,852.50$ & .7 \\
Laterality & $55,058.01$ & 1 & $55,058.01$ & .00004 \\
Error (Laterality) & $68,575.68$ & 8 & 857.21 & .23 \\
Familiarity $\times$ Laterality & 837.21 & 1 & 837.21 & .96 \\
Error (Familiarity $\times$ Laterality) & $7,015.06$ & 8 & 876.88 & .35 \\
\hline
\end{tabular}




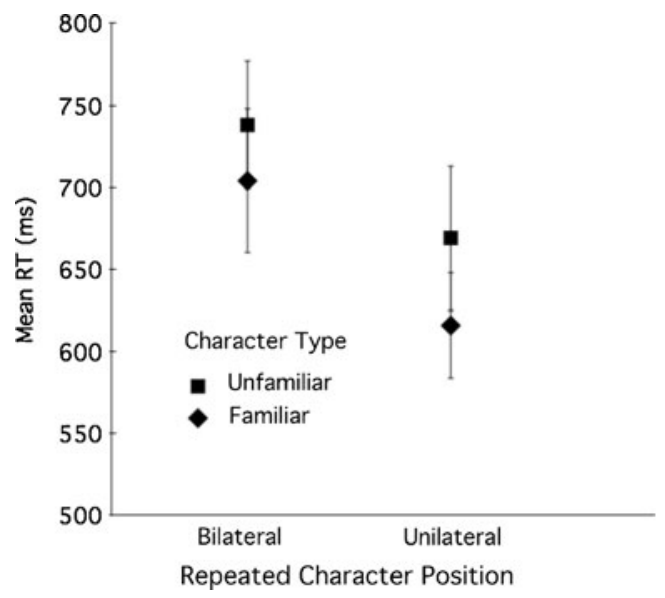

Fig. 4 Mean reaction times as a function of repeated-character laterality for familiar and unfamiliar characters $(n=9)$. For familiar characters, unilateral repetitions were detected $88 \mathrm{~ms}$ faster than bilateral repetitions $(p<.0001)$. For unfamiliar characters, unilateral repetitions were detected $69 \mathrm{~ms}$ faster than bilateral repetitions $(p<.002)$

this experiment. All of the subjects were native English speakers and reported no knowledge of the Arabic or katakana alphabets. All had normal or corrected-to-normal vision.

Stimuli The stimuli were characters from the Western, Arabic, and katakana character sets and were identical to those used in Experiment 1.

Apparatus All stimuli were presented on a Macintosh computer with a 25 -in. Apple Studio Display monitor, at a resolution of $1,280 \times 1,024$ and a refresh rate of $75 \mathrm{~Hz}$, with E-Prime software.

Procedure The procedure for Experiment 2 was identical to that of Experiment 1, with the following exception. In this experiment, the stimuli were presented for $150 \mathrm{~ms}$. Subjects performed repetition detection on one block each of Western, katakana, and Arabic characters. The block order was counterbalanced across subjects, with each subject performing 20 practice and 480 experimental trials in each block.

\section{Results}

Figure 5 shows the mean reaction times for detecting repetitions of Western, Arabic, and katakana characters. The mean reaction time data and miss rates were subjected to a $3 \times 2$ repeated measures ANOVA, with Character Type (Western, katakana, or Arabic) and Laterality (unilateral or bilateral) as the factors (Table 5). As in Experiment 1, we found a significant main effect of laterality $[F(1,11)=$ 20.29, $p<.001$, two-tailed]. Neither the main effect of character nor the Laterality $\times$ Character interaction was significant. For Western characters, unilateral repetitions were

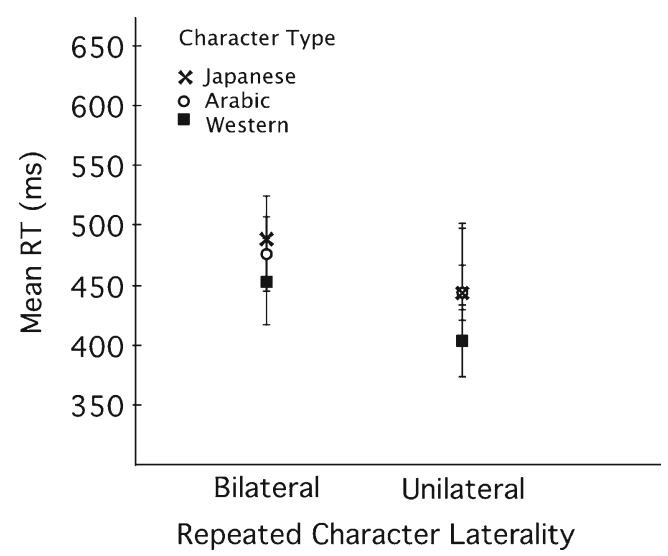

Fig. 5 Mean reaction time for native English speakers for repetition detection of Western, Arabic, and katakana characters $(n=12)$. The error bars indicate $\pm 1 S E M$

detected $49 \mathrm{~ms}$ faster than bilateral repetitions $[t(11)=3.94$, $p<.002$, two-tailed]; for katakana characters, unilateral repetitions were detected $41 \mathrm{~ms}$ faster than bilateral repetitions $[t(11)=3.75, p<.003$, two-tailed $]$; and for Arabic characters, the 32-ms reaction time difference for unilateral versus bilateral repetitions did not reach two-tailed significance $[t(11)=$ $1.85, p<.09]$.

For the miss rate analysis, the $3 \times 2$ ANOVA revealed a main effect of laterality $[F(1,1)=18.56, p<.001]$ on miss rates. Neither the main effect of character nor the Laterality $\times$ Character interaction was significant. For Western characters, the subjects made significantly more misses for bilateral trials [bilateral mean $5 \%$, unilateral mean $3 \% ; t(11)=4.12, p<$ .002 , two-tailed]. For katakana characters, the subjects also made significantly more errors on bilateral trials [bilateral mean $6 \%$, unilateral mean $3 \% ; t(11)=2.78, p<.02$, twotailed]. And finally, for Arabic characters the subjects also made significantly more misses for bilateral trials [bilateral mean $7 \%$, unilateral mean $4 \% ; t(11)=3.80, p<.003$, twotailed]. The target-absent mean reaction times were $484 \mathrm{~ms}$ for Western characters, $561 \mathrm{~ms}$ for Arabic characters, and $574 \mathrm{~ms}$ for katakana characters. Post-hoc testing revealed that these differences were not significant.

\section{Discussion}

In Experiment 2, we presented the target stimuli for $150 \mathrm{~ms}$, and we once again found a significant unilateral field advantage that did not interact with stimulus familiarity. In this experiment, all of the subjects were native English speakers and were unfamiliar with both Arabic and katakana characters, yet there was no interaction of character type and laterality for either the reaction time or the error data. The subjects were faster and more accurate when detecting unilateral repetitions, regardless of familiarity; additional exposure time did not allow familiarity effects to manifest. 
Table 5 Experiment 2: Repeated measures $2 \times 3$ ANOVA - laterality (bilateral, unilateral) $\times$ character type (Western, Japanese, katakana)

\begin{tabular}{lllll}
\hline Source & Sum of Squares & $d f$ & Mean Square & $F$ \\
\hline Laterality & $31,823.93$ & 1 & $31,823.93$ & 20.29 \\
Error (Laterality) & $17,250.213$ & 11 & $1,568.201$ & .001 \\
Character & $19,771.970$ & 2 & $9,885.99$ & .45 \\
Error (Character) & $488,705.25$ & 22 & $22,213.88$ & .65 \\
Laterality $\times$ Character & 934.71 & 2 & 467.37 & .47 \\
Error (Laterality $\times$ Character) & $21,944.67$ & 22 & 997.49 & .63 \\
\hline
\end{tabular}

\section{General discussion}

We have proposed previously that the unilateral field advantage for detecting repetitions stems from a general, low-level perceptual-grouping benefit (Butcher \& Cavanagh, 2008). We found that subjects were faster detecting unilateral repetitions of stimuli processed in both the ventral (letters, colors, and sizes) and dorsal (common motion paths) visual pathways (Butcher \& Cavanagh, 2008). We posited that the unilateral field advantage suggests that the neural locus of this grouping benefit arises from efficient processing in early visual areas, where receptive fields are limited to the contralateral field (Abel, O'Brien, \& Olavarria, 2000; Clarke \& Miklossy, 1990; Kennedy \& Dehay, 1988). In higher visual areas, receptive fields often or always cross the vertical meridian (DeYoe et al., 1996; Engel, Glover, \& Wandell, 1997; Huk, Dougherty, \& Heeger, 2002; Tootell, Mendola, Hadjikhani, Liu, \& Dale, 1998).

Here, we again found an effect of laterality for detecting repetitions, but no effect of familiarity. This finding supports our claim that this benefit is low-level and arises in early visual areas that do not have immediate access to information in visual memory. Hierarchical models of visual perception have historically considered perceptual grouping as an early, low-level process (Neisser, 1967; Treisman, 1986, 1988; Treisman \& Gelade, 1980; Treisman \& Gormican, 1988) that provides inputs for higher levels of processing, such as visual attention and object recognition. In this view, perceptual grouping is a bottom-up process, relatively uninfluenced by higher-level top-down factors, such as familiarity. Our results and interpretation of them are consistent with this view.

The lack of a familiarity effect here runs contrary to previously reported results from target detection and visual search tasks. Wang et al. (1994) found that familiar distractor letters produced efficient search for unfamiliar target letters, and suggested that the familiarity of individual items is extracted rapidly in display search. In their displays, subjects searched for a either a mirror-reversed " $\mathrm{N}$ " in an array of normally oriented "N"s or vice versa. The low-level features of both targets and distractors were comparable. However, only the search for the mirror-reversed "N" produced a parallel Reaction Time $\times$ Set Size function, the hallmark of efficient search. In our experiment, subjects were presented with displays of either all familiar or all novel characters, but we found no effect of familiarity for repetition detection. According to Wang et al., proficient search for the unfamiliar target results from efficient grouping of the familiar distractors. Their data suggest that this type of grouping does not occur for unfamiliar distractors, producing search slopes of $43 \mathrm{~ms} /$ item. How can we bring this finding in line with our results, which suggest that both familiar and unfamiliar items can be grouped efficiently within a hemifield?

In our display, all of the items were either familiar or unfamiliar. It may be that familiarity information was in fact extracted rapidly from our displays. However, this information may only be useful in reaction time performance when stimuli with different levels of familiarity are presented in the same display. Recall that Malinowski and Hübner (2001) found that German subjects were faster at finding an unfamiliar mirror-reversed "N" among "N"s. However, Slavic subjects, who were familiar with both characters, showed no difference in performance as a function of which character was the target versus the distractor. Familiarity might act as a differential variable that is useful for signaling the presence of a novel region, but is of limited task value when all of the items possess the same level. Future experiments of repetition detection in which the targets and distractors possess graded levels of familiarity would be useful for assessing this hypothesis.

In all of our experiments, the targets consisted of repetitions that allowed the target to form a homogeneous twoitem group among heterogeneous distractors. The targets were thus defined by information redundancy. It has previously been established that keypress reaction times are faster for redundant than for similar signals. This phenomenon, know as the redundant-target effect (RTE), occurs when reaction times for a double stimulus presentation are compared with those of single stimulus presentations (Hershenson, 1962; Meijers \& Eijkman, 1977; Miniussi, Girelli, \& Marzi, 1998; Raab, 1962). In our experiments, repeated targets were never compared with single targets, and the targets were defined only by their redundancy. However, our data notably indicate that redundant targets presented unilaterally versus 
bilaterally do not elicit equivalent behavioral performance, suggesting that the RTE might also show a unilateral advantage if it were tested in future single-versus-dual-target studies of the phenomenon.

While familiarity is known to provide advantages in visual search tasks (Rauschenberger \& Chu, 2006; Shen \& Reingold, 2001; Wang et al., 1994), in other instances it has little to no effect. Pashler (1988), for instance, found that familiarity did not play a role in change blindness. In Pashler's experiments, subjects were briefly presented with two sequential arrays of letters, and their task was to detect the presence of a change in one of the letters. The results indicated that subjects were poor at detecting these changes at interstimulus intervals of $67 \mathrm{~ms}$ or greater. To investigate the effect of familiarity, Pashler compared subjects' change detection performance with familiar alphanumeric characters to performance with a character set composed of those same characters reflected with respect to their horizontal axis (Pashler, 1988, Exp. 3). Reflection had no effect on change detection accuracy. Pashler concluded that this lack of a familiarity effect revealed that the visual short-term memory system, which was used to store information for comparison of the two arrays, does not depend on item recognition or familiarity. The lack of a familiarity benefit for change detection has also been shown for other novel stimuli (e.g., polygons; Chen, Eng, \& Jiang, 2006), even when subjects gain familiarity with the stimuli by completing over 5,000 trials over the course of 10 sessions (Eng, Chen, \& Jiang, 2005). Our task did not require visual short-term memory storage for sequential comparison, in the manner of a change detection task. However, we found that efficient detection of unilateral repetitions did not require item familiarity.

While we have proposed that the unilateral field advantage results from efficient within-field perceptual grouping (Butcher \& Cavanagh, 2008), other theories have been suggested to account for such within-field processing benefits. Marsolek, Nicholas, and Andresen (2002) found that subjects were more accurate when reporting that two letters, which were similar exemplars of the same physical category (e.g., "a" and "a"), matched when the letters were presented within a visual field. This pattern reversed when subjects were asked to determine whether two letters were both members of the same abstract category (e.g., "A" and "a"). Marsolek et al. and others (Banich \& Belger, 1990) have generally proposed that differing performance for withinand across-field trials reflects a trade-off between the benefit of distributing information across visual fields, and thus across hemispheres, with the cost of interhemispheric transfer for across-field presentations. More specifically, Marsolek et al. proposed that the difference in visual field effects as a function of the identity match type required - abstract or specific - indicates that these types of information are processed interhemispherically via dissociable subsystems.
Does our efficient-within-field-grouping explanation of the unilateral field advantage contradict Marsolek et al.'s (2002) theory? We cannot specifically address the idea of separate subsystems for abstract and specific exemplar matches, as here we have only examined the latter. Do our results indicate efficient within-field grouping, or are we seeing the results of poor across-field interhemispheric transfer for a specific exemplar subsystem, leading to less efficient detection of acrossfield repetitions? Are these options mutually exclusive? Further studies will be needed to investigate these questions.

In our experiments, we used a four-element paradigm with equidistant spacing between unilateral and bilateral repetitions. Marsolek et al. (2002) used a rectangular arrangement with unequal sides: Bilateral stimuli were spaced farther apart than were elements in the same visual field. In our task, repetitions occurred on the vertices of an invisible square, while for Marsolek et al. they occurred on a diagonal. Further studies that reduce the methodological disparity between these experimental designs would enable a more direct comparison of these interpretations of the UFA.

While they did not specifically address the question of familiarity, Hayes et al. (2010) explored the effects of perceptual similarity and task demands on the UFA for repetition detection using a four-element paradigm similar to our own. In separate experiments, they varied the similarity of the repeated elements (e.g., repeated objects from different viewpoints or of different scales, or repeated letters that varied in color) and the difficulty of detecting the repetition (e.g., detecting the repetition of a color target with distractors that were different shades of the same color). Hayes et al. suggested that if perceptual grouping was responsible for the UFA, decreasing the strength of the groups should decrease the magnitude of the UFA. However, they found the opposite pattern of results for accuracy: The manipulations that reduced perceptual similarity increased accuracy for detecting unilateral versus bilateral repetitions. It is important to note that they did not find differences in reaction times as a function of their similarity manipulations. Thus, the efficiency of detection remained the same as perceptual similarity varied. This reaction time data do not conflict with our efficientwithin-field-grouping interpretation, since in both their study and our own, unilateral repetitions were detected faster than bilateral repetitions. Rather, Hayes et al. extended the types of groups that produce this pattern of advantages. Future studies should seek to specifically clarify the role of similarity in both reaction times and accuracy for the UFA and should address Hayes et al.'s counterintuitive accuracy finding.

In conclusion, we extended our previous results on the unilateral field advantage: Subjects are faster reporting the presence of a repetition when it is presented unilaterally rather than bilaterally. Familiarity does not interact with this effect, since subjects are faster at detecting both familiar and unfamiliar repetitions when they are presented in one visual 
field versus two. We have proposed that physical repetitions may be processed by detecting the group formed by the repetition, and we now suggest that this grouping process occurs at an early level of processing, prior to the levels at which familiarity affects coding.

Author note S.J.B. is now at the Department of Psychology, Hamilton College; P.C. is now at the Institut de Psychologie, Université Paris Descartes. This research was supported by an NSF Graduate Fellowship to S.J.B.

\section{References}

Abel, P. L., O’Brien, B. J., \& Olavarria, J. F. (2000). Organization of callosal linkages in visual area $\mathrm{V} 2$ of macaque monkey. The Journal of Comparative Neurology, 428, 278-293. doi:10.1002/ 1096-9861(20001211)428:2<278::AID-CNE7>3.0.CO;2-R.

Banich, M. T., \& Belger, A. (1990). Interhemispheric interaction: How do the hemispheres divide and conquer a task? Cortex, 26, 77-94.

Bouvrie, J. V., \& Sinha, P. (2007). Object concept learning: Observations in congenitally blind children and a computational model. Neurocomputing, 70, 2218-2233. doi:10.1016/j.neucom.2006.01.035

Butcher, S. J., \& Cavanagh, P. (2008). A unilateral field advantage for detecting repeated elements. Attention, Perception, \& Psychophysics, 70, 714-724. doi:10.3758/PP.70.4.714

Cavanagh, J. P., \& Parkman, J. M. (1972). Search processes for detecting repeated items in a visual display. Perception \& Psychophysics, 11, 43-45. doi:10.3758/BF03212681

Chen, D., Eng, H. Y., \& Jiang, Y. (2006). Visual working memory for trained and novel polygons. Visual Cognition, 14, 37-54. doi:10.1080/13506280544000282

Clarke, S., \& Miklossy, J. (1990). Occipital cortex in man: Organization of callosal connections, related myelo- and cytoarchitecture, and putative boundaries of functional visual areas. The Journal of Comparative Neurology, 298, 188-214. doi:10.1002/ cne.902980205

Comtois, R. (2003). Vision Shell PPC [Software libraries]. Watertown, MA: Author.

DeYoe, E. A., Carman, G. J., Bandettini, P., Glickman, S., Wieser, J., Cox, R., . . . Neitz, J. (1996). Mapping striate and extrastriate visual areas in human cerebral cortex. Proceeding of the National Academy of Science, 93, 2382-2386. doi:10.1073/pnas.93.6.2382

Eng, H. Y., Chen, D., \& Jiang, Y. (2005). Visual working memory for simple and complex visual stimuli. Psychonomic Bulletin \& Review, 12, 1127-1133. doi:10.3758/BF03206454

Engel, S. A., Glover, G. H., \& Wandell, B. A. (1997). Retinotopic organization in the human visual cortex and spatial precision of functional MRI. Cerebral Cortex, 7, 181-192. doi:10.1093/cercor/7.2.181

Fine, I., Wade, A. R., Brewer, A. A., May, M. G., Goodman, D. F., Boynton, G. M., . . . MacLeod, D. I. A. (2003). Long-term deprivation affects visual perception and cortex. Nature Neuroscience, 6, 915-916. doi:10.1038/nn1102

Hayes, M. T., Swallow, K. M., \& Jiang, Y. V. (2010). The unilateral field advantage in repetition detection: Effects of perceptual grouping and task demands. Attention, Perception, \& Psychophysics, 72, 583-590. doi:10.3758/APP.72.3.583

Hershenson, M. (1962). Reaction time as a measure of intersensory facilitation. Journal of Experimental Psychology, 63, 289-293. doi: $10.1037 / \mathrm{h} 0039516$

Huk, A. C., Dougherty, R. F., \& Heeger, D. J. (2002). Retinotopy and functional subdivision of human areas MT and MST. Journal of Neuroscience, 22, 7195-7205.
Kennedy, H., \& Dehay, C. (1988). Functional implications of the anatomical organization of the callosal projections of visual areas $\mathrm{V} 1$ and V2 in the macaque monkey. Behavioral Brain Research, 29, 225-36. doi:10.1016/0166-4328(88)90027-7

Malinowski, P., \& Hübner, R. (2001). The effect of familiarity on visualsearch performance: Evidence for learned basic features. Perception \& Psychophysics, 63, 458-463. doi:10.3758/BF03194412

Marsolek, C. J., Nicholas, C. D., \& Andresen, D. R. (2002). Interhemispheric communication of abstract and specific visual-form information. Neuropsychologia, 40, 1983-1999. doi:10.1016/S00283932(02)00065-9

Meijers, L. M. M., \& Eijkman, E. G. J. (1977). Distributions of simple RT with single and double stimuli. Perception \& Psychophysics, 22, 41-48. doi:10.3758/BF03206078

Miniussi, C., Girelli, M., \& Marzi, C. A. (1998). Neural site of the redundant target effect: Electrophysiological evidence. Journal of Cognitive Neuroscience, 10, 216-230. doi:10.1162/089892998562663

Neisser, U. (1967). Cognitive psychology. New York, NY: AppletonCentury-Crofts.

Ostrovsky, Y., Andalman, A., \& Sinha, P. (2007). Vision following extended congenital blindness. Psychological Science, 17(12), 1009-1014. doi:10.1111/j.1467-9280.2006.01827.x

Pashler, H. (1988). Familiarity and visual change detection. Perception \& Psychophysics, 44, 369-378. doi:10.3758/BF03210419

Raab, D. (1962). Statistical facilitation of simple reaction times. Transactions of the New York Academy of Sciences, 24, 574-590.

Rauschenberger, R., \& Chu, H. (2006). The effect of stimulus rotation and familiarity in visual search. Perception \& Psychophysics, 68 (5), 770-775. doi:10.3758/BF03193700

Rauschenberger, R., \& Yantis, S. (2006). Perceptual encoding efficiency in visual search. Journal of Experimental Psychology. General, 135 (1), 116-131. doi:10.1037/0096-3445.135.1.116

Shen, J., \& Reingold, E. M. (2001). Visual search asymmetry: The influence of stimulus familiarity and low-level features. Perception \& Psychophysics, 63, 464-475. doi:10.3758/BF03194413

Tootell, R. B. H., Mendola, J. D., Hadjikhani, N. K., Liu, A. K., \& Dale, A. M. (1998). The representation of the ipsilateral visual field in human cerebral cortex. Proceedings of the National Academy of Sciences, 95, 818-824.

Treisman, A. (1986). Features and objects in visual processing. Scientific American, 254(11), 114-125. doi:10.1038/scientificamerican1186$114 \mathrm{~B}$

Treisman, A. (1988). Features and objects: The Fourteenth Bartlett Memorial Lecture. Quarterly Journal of Experimental Psychology, 40A(2), 201-237. doi:10.1080/02724988843000104

Treisman, A. M., \& Gelade, G. (1980). A feature-integration theory of attention. Cognitive Psychology, 12, 97-136. doi:10.1016/00100285(80)90005-5

Treisman, A., \& Gormican, S. (1988). Feature analysis in early vision: Evidence from search asymmetries. Psychological Review, 95, 15-48. doi:10.1037/0033-295X.95.1.15

Treisman, A., \& Souther, J. (1985). Search asymmetry: A diagnostic for preattentive processing of separable features. Journal of Experimental Psychology. General, 114, 285-310. doi:10.1037/ 0096-3445.114.3.285

Wang, Q., Cavanagh, P., \& Green, M. (1994). Familiarity and pop-out in visual search. Perception \& Psychophysics, 56, 495-500. doi:10.3758/BF03206946

Weissman, D. H., \& Banich, M. T. (1999). Global-local interference modulated by communication between the hemispheres. Journal of Experimental Psychology. General, 128, 283-308. doi:10.1037/ 0096-3445.128.3.283

Weissman, D. H., Banich, M. T., \& Puente, E. I. (2001). An unbalanced distribution of inputs across the hemispheres facilitates inter-hemispheric interaction. Journal of the International Neuropsychological Society, 6, 313-321. 